\title{
A review on brain-derived neurotrophic factor and its role in depression and anxiety
}

\author{
Vlad Teodor Iacob, Matei Palimariciuc, \\ Vasile Chiriță, Roxana Chiriță
}

\begin{abstract}
Vlad Teodor Iacob - M.D., junior psychiatrist, "Socola” Institute of Psychiatry, Iași, Romania Matei Palimariciuc - M.D., PhD student, Assistant professor, "Grigore T. Popa" University of Medicine and Pharmacy Iași, Romania, junior psychiatrist "Socola" Institute of Psychiatry, Iași, Romania Vasile Chiriță - Professor, Academician, PhD, Psychiatrist, Honorary Member of Romanian Academy

Roxana Chiriță - M.D. PhD, Professor of Psychiatry "Grigore T. Popa" University of Medicine and Pharmacy Iași, Romania, senior psychiatrist "Socola" Institute of Psychiatry, Iași, Romania
\end{abstract}

\begin{abstract}
Brain-derived neurotrophic factor (BDNF) is a well-deliberated growth factor that serves many purposes throughout the central nervous system (CNS) and has been implicated in various psychiatric and neurological disorders. This article reviews the current understanding and future directions in BDNF-related research in the CNS, with an emphasis on its relation to depression and anxiety-related disorders.
\end{abstract}

\section{KEYWORDS:}

BDNF, neurotrophin, depression, anxiety.

\section{INTRODUCTION}

Mood and anxiety disorders are arguably amongst the most disabling of all medical disorders. They frequently appear together and early in life, run a chronic course and adversely affect the prognosis of other medical illnesses (1). Understanding the pathophysiology of these disorders is crucial in the effort to better predict or prevent their onset, to develop new treatments, and to better adapt each treatment to each individual (2). Brain-derived neurotrophic factor (BDNF) is a well-studied growth factor that

serves many functions within the central nervous system (CNS) and has been implicated in several psychiatric disorders, such as autism, intellectual disability, schizophrenia, and the development of anxiety and mood disorders, such as depression and its treatment (3).

BDNF FROM A MORPHOFUNCTIONAL STANDPOINT 
BDNF is a member of the neurotrophin family, including nerve growth factor (NGF), neurotrophin-3 (NT3) and neurotrophin-4 (NT4). For almost 40 years, research has been conducted on BDNF, and while it has been fruitful in many fields, we have yet to fully understand it and use it to its full potential. Through dendritic arborization and synaptic consolidation, BDNF mediates, amongst others, neuronal development, synaptic plasticity and long-term potentiation in the CNS (4, 5, 6, 7, 8). BDNF also influences learning and memory $(9,10,11)$, and modulates hypothalamic metabolic function $(12,13)$. It is secreted by neurons and peripheral cells, including leukocytes (14), endothelial cells (15), and platelets $(16,17)$, and passes the blood-brain barrier (18), albeit poorly (4).

Although the matter is still debatable, it seems that there are two forms of extracellular BDNF: proBDNF and mature BDNF (mBDNF). After its release from neurons, the pro-domain of pro-BDNF is proteolytically cleaved by plasmin or metalloproteinases, resulting mBDNF $(19,20)$. Recent studies suggest that the mature form of BDNF is predominant and of greatest physiological relevance in the adult brain (21.22). Regarding receptor binding affinities, BDNF binds with high specificity to the tropomyosin receptor kinase $\mathrm{B}$ (TrkB) and the low-affinity neurotrophin receptor p75 (p75NTR). While TrkB is widely expressed throughout the adult brain, including the cortex, hippocampus, and multiple brainstem and spinal cord nuclei (23), the expression of p75NTR is restricted in adulthood to basal forebrain cholinergic neurons and relatively few cortical neurons (24). Furthermore, mBDNF binds with great affinity to TrkB, whereas pro-BDNF binds with a higher affinity to p75NTR (19). This distinction in BDNF isoform binding is relevant because TrkB predominantly supports neuronal survival and expression of several genes (25), while separate p75NTR activations result in pro-apoptotic signalling (26).

\section{BDNF AND ITS FUNCTION IN MOOD AND ANXIETY DISORDERS}

A number of individual studies and later meta-analyses in the last few decades have reported perturbations in BDNF expression in both depression and anxiety, and its return to normal levels after various treatments for depression, including antidepressants $(27,28$, 29).Reduced BDNF levels have been found in the postmortem samples of brains of depressed patients and of suicide victims, many of which suffer from severe depression (29). Furthermore, levels of its receptor, TrkB, have also been reported to be downregulated in the brain of depressed patients (30) and the levels of active, phosphorylated form of TrkB are reduced in suicide victims (31), indicating reduced BDNF signalling through TrkB in depression. On the other hand, increased levels of mRNA for the p75NTR neurotrophin receptor have been reported in the brains of suicide victims (32). In the adult brain, upon neuronal trauma, p75NTR expression is increased in cortical and hippocampal neurons and as previously mentioned, p75NTR mediates degeneration and death of injured neurons (26, 29). Increase in p75NTR levels in suicide victims suggests that severe psychological stress, such as leading to suicide, may act similar to brain trauma. p75NTR signalling may promote synaptic depression and pruning and other "antitrophic" effects that could at least partially explain the pathophysiology of depression (29).

Regarding treatment, conventional antidepressants, such as selective serotonin reuptake inhibitors (SSRIs) or tricyclic antidepressants (TCAs), mediate their 
Bulletin of Integrative Psychiatry O New Series OSeptember 2020 ○ Year XXVI ONo. 3 (86)/81

\%

antidepressant effect by modulating extracellular levels of monoamines, mainly serotonin or norepinephrine. It is thought that these drugs enhance the extracellular levels of monoamines rather quickly, within hours.

However, the antidepressant response is delayed and typically requires weeks of treatment before the onset of therapeutic actions $(33,34)$. Therefore, other mechanisms relating to the enhancement of extracellular level of monoamines are believed to mediate the antidepressant response (4). In this context, BDNF has been linked to the mechanism of action of antidepressants. The first studies to implicate BDNF in antidepressant responses reported that conventional antidepressant drugs, as well as electroconvulsive therapy (ECT), enhanced BDNF and TrkB mRNA expression in the hippocampus and cortical regions in a time span similar to the onset of therapeutic actions of antidepressants. Subsequent studies took a more direct approach in their research by infusing BDNF protein directly into the midbrain of rodents, observing an antidepressant-like effect (35). Further work showed that a single bilateral infusion of a low dose of BDNF directly into the dentate gyrus (DG) or CA3, but not CA1 region of the hippocampus was sufficient to induce an antidepressant-like effect within three days (5), suggesting that these regions may be critical to understanding the antidepressant effect in connection to BDNF. Moreover, this latter study noted that BDNF produced an antidepressant effect for up to 10 days after infusion, well past the time frame for the degradation of the protein, suggesting that BDNF may be triggering a sustained plasticity mechanism to mediate the long-term antidepressant effects. Its theory states that the antidepressant effects of BDNF are mediated by TrkB activation of mitogenactivated protein kinase kinase (MEK), acting through the extracellular signal-regulated pathway (5). In a separate study, peripheral, subcutaneous injections of BDNF were reported to produce antidepressant and anxiolytic behavioural responses in animal models, prevent depression-related effects of chronic stress, and an increase to the survival rate of newborn neurons in the hippocampus and prefrontal cortex (36). However, the exact mechanism of this conclusion remains to be explained, as BDNF is poorly transported across the blood-brain barrier, leaving it unclear as to how peripheral administration mediates the aforementioned effects (4).

Further evidence supporting the involvement of BDNF signalling due to the effects of antidepressant drugs has emerged from studies examining the high-affinity BDNF receptor, TrkB. Antidepressants require functional $\operatorname{TrkB}$ receptors to mediate antidepressant-like responses in preclinical models (37, 38). Interestingly, long-term antidepressant treatment increases the phosphorylation of the TrkB receptor, thus increasing its activity in the hippocampus and anterior cingulate cortex suggesting that BDNF-TrkB signalling in these regions may be of great importance in mediating antidepressant effects (37). The lastmentioned study has also concluded that conventional antidepressants necessitate monoamines to affect BDNF intracellular signalling as monoamine depletion prevented the antidepressant-induced activation of TrkB (37).

While there is strong data that supports the fact that BDNF in the hippocampus and prefrontal cortex is involved in mediating antidepressant responses, other brain regions have also been involved in antidepressant responses. For example, BDNF signalling in the ventral tegmental area (VTA) to nucleus accumbens pathway appears to be producing 
effects that are in many ways opposite to the effects of BDNF in the hippocampus (39). BDNF injected directly into the VTA induces depression-like behaviour and, contrarily, blocking BDNF signalling in the nucleus accumbens, a brain region which receives afferents from the VTA, induces an antidepressant-like effect (40). BDNF signalling in this region has also been found to be essential for the development of the molecular and behavioural manifestations of stress $(41,42)$, hence further proving the involvement of BDNF in the mesolimbic pathway in the expression of at least a subgroup of the depressive symptoms. Another brain region that has been implicated in mood disorders is the lateral habenula, although it remains unclear if BDNF in this region has any connection to antidepressant responses or depression-related behaviour (4). As a result, BDNF-TrkB signalling in the hippocampus seems to be a vital component of antidepressant responses to clinically used antidepressants, at least in preclinical animal models. Nonetheless, the involvement of the hippocampus does not exclude the involvement of other brain regions, as, without a doubt, distinct neural circuits are required for antidepressant responses. Although, the requirement for BDNF in the hippocampus in mediating antidepressant responses is the first step to fully understand the neural circuitry involved in this process (4).

\section{THERAPEUTIC POTENTIAL AND THE DIFFICULTY OF DELIVERY TO THE CNS}

Even amongst the nervous system growth factors, BDNF has emerged as a leading candidate of interest as a potential neuroprotective and functionally restorative treatment in various psychiatric and neurological disorders, including amyotrophic lateral sclerosis (ALS), peripheral neuropathy,
Alzheimer's disease and Parkinson's disease (28).

As previously stated, a considerable number of studies reported alterations in BDNF expression in depressive and anxiety-related disorders. Patients who suffer from depression exhibit specific reductions in hippocampal volume, and in hippocampal and serum BDNF levels. Antidepressants reverse reductions in hippocampal volume, and several antidepressant therapies have been reported to normalize serum BDNF levels. Given the fact that BDNF is important in maintaining hippocampal dendritic morphology and synaptic function (4-8), it may contribute to the reversal of hippocampal atrophy due to the effects of antidepressants, although other factors may also be likely involved, such as activity and exercise (28).

The main challenge in the field of growth factor therapy is the drug delivery system to the CNS. BDNF is a reasonably sized and charged protein, which accounts for the fact that a minimal amount of BDNF crosses the blood-brain barrier via peripheral administration (28). In order to reach neurons from the brain or spinal cord, BDNF must be administered directly into the CNS. However, when administered intraventricularly or intrathecally, BDNF infusion results in little penetration of the brain or spinal cord parenchyma beyond the most superficial layers (43). Moreover, when growth factors do "spread" through the nervous system after intrathecal administration, they cause intolerable adverse effects over long periods of time. These adverse effects include weight loss, dysaesthesia and migration and/or proliferation of Schwann cells in the subpial space (44-47). Another important observation in the study of growth factor therapies is the need for extended periods of treatment, depending on which disorder is being treated. 
Thus, the ideal delivery system of growth factors to the nervous system must achieve concentrations at precise sites to limit the spread to distant sites and, as such, prevent adverse effects as much as possible. Two potential approaches are in the running for a safe delivery system of growth factors such as BDNF to the nervous system: intraparenchymal protein infusion (48-50) and gene delivery using viral vectors (51), both of which require further development (28).

\section{DISCUSSION}

While there is still much controversy over the relation between BDNF and anxiety in animals and humans (52-56), there is a vast amount of knowledge and evidence that strongly implicates BDNF in the pathophysiology of depression, and many other medical conditions. These findings lead to several potentially translational possibilities. To start with, BDNF has great potential for its use as a biomarker, for diagnosing mood disorders and monitoring their treatment response. There is also the therapeutic potential of BDNF for treating various psychiatric and neurological disorders, with ongoing research fiercely trying to solve the drug delivery system challenge through different approaches: by enhancing the accuracy of vector and growth factor targeting to the brain using real-time MRI during treatment (57); by improving gene therapy vectors to achieve persistent, safe and regulatable growth factor expression in the CNS (58); and by developing peptide mimetics of growth factors with specificity for degenerating cell systems (59).

Nonetheless, we still have a long way to go until we can fully understand its mechanism of action and apply that information to successfully construct and customise new treatment options. There is a great deal of work needed in delineating the regional specificity of BDNF TrkB signalling, with the hippocampus and its involvement in mediating antidepressant responses being the first step for fully understanding the neural circuitry involved in this process.

\section{CONCLUSIONS}

The data reviewed above suggests a close relationship between BDNF signalling, neuronal plasticity and depression and its treatment, with ongoing research focused on applying BDNF as a viable biomarker and treatment option.

\section{ACKNOWLEDGEMENTS AND DISCLOSURES}

The authors declare that they have no potential conflict of interest to disclose.

\section{REFERENCES}

1. Kalaria RN, Maestre GE, Arizaga R, Friedland RP, et al.2008.Alzheimer's disease and vascular dementia in developing countries: prevalence, management, and risk factors. Lancet Neurol 7:812-826.

2. Guest PC, Reviews on Biomarker Studies in Psychiatric and Neurodegenerative Disorders. Department of Biochemistry and Tissue Biology Institute of Biology University of Campinas (UNICAMP) Campinas, Brazil.

3. Autry AE, Monteggia LM. Brain-derived neurotrophic factor and neuro-psychiatric disorders. Pharmacol. Rev. 2012; 64(2):238-258.

4. Björkholm C, Monteggia LM. BDNF-a key transducer of antidepressant effects.Neuropharmacology. 2016;102:7279.

5. Shirayama Y, Chen AC, Nakagawa S, Russell DS, et al. Brain-derived neurotrophic factor produces antidepressant effects in behavioral models of depression. J. Neurosci.2002; 22(8):3251-3261.

6. Polyakova M, Stuke K, Schuemberg K, Mueller K, et al. BDNF as a biomarker for successful treatment of mood disorders: a systematic \& quantitative meta-analysis.J Affect Disord. 2015;174:432-440. 


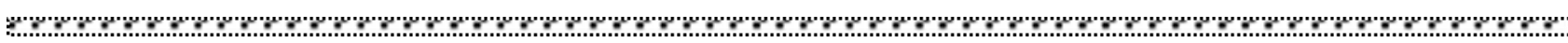
7. Figurov A., Pozzo-Miller L. D., Olafsson, P., et al. Regulation of synaptic responses to high-frequency stimulation and LTP by neurotrophins in the hippocampus. Nature. 1996; 381, 706-709.

8. Kang H., Schuman EM. Long-lasting neurotrophin-induced enhancement of synaptic transmission in the adult hippocampus. Science.1995;267, 1658-1662.

9. Yamada K, Mizuno M, Nabeshima T. Role for brain-derived neurotrophic factor in learning and memory. Life Sci. 2002;70, 735-744.

10. Egan MF, Kojima M, Callicott JH, et al. The BDNF val66met polymorphism affects activity-dependent secretion of BDNF and human memory and hippocampal function. Cell. 2003;112, 257-269.

11. Cathomas F, Vogler C, Euler-Sigmund JC, de Quervain DJ, et al. Fine-mapping of the brain-derived neurotrophic factor (BDNF) gene supports an association of the Val66Met polymorphism with episodic memory. Int. J. Neuropsychopharmacol. 2010;13, 975-980.

12. Xu B, Goulding EH, Zang K, et al. Brain-derived neurotrophic factor regulates energy balance downstream of melanocortin-4 receptor. Nat Neurosci. 2003;6(7):736-742.

13. Cao L, Lin EJ, Cahill MC, Wang C, Liu X, During MJ. Molecular therapy of obesity and diabetes by a physiological autoregulatory approach. Nat Med. 2009;15(4):447-454.

14. Edling AE, Nanavati T, Johnson JM, Tuohy VK. Human and murine lymphocyte neurotrophin expression is confined to B cells. J Neurosci Res. 2004;77(5):709-717.

15. Nakahashi T, Fujimura H, Altar CA, et al. Vascular endothelial cells synthesize and secrete brain-derived neurotrophic factor. FEBS Lett. 2000;470(2):113-117.

16. Yamamoto H, Gurney ME. Human platelets contain brain-derived neurotrophic factor. J Neurosci. 1990;10(11):3469-3478.

17. Fujimura H, Altar CA, Chen R, et al. Brain-derived neurotrophic factor is stored in human platelets and released by agonist stimulation. Thromb Haemost. 2002;87(4):728-734.

18. Pan W, Banks WA, Fasold MB, Bluth J, et al. Transport of brain-derived neurotrophic factor across the blood-brain barrier. Neuropharmacology.1998;37(12):1553-1561.

19. Lee R, Kermani P, Teng KK, Hempstead BL. Regulation of cell survival by secreted proneurotrophins. Science. 2001;294(5548):1945-1948.

20. Nikoletopoulou V, Lickert H, Frade JM, et al. Neurotrophin receptors TrkA and TrkC cause neuronal death whereas TrkB does not. Nature. 2010;467(7311):59-63.

21. Rauskolb S, Zagrebelsky M, Dreznjak A, et al. Global deprivation of brain-derived neurotrophic factor in the CNS reveals an area-specific requirement for dendritic growth. J Neurosci. 2010;30(5):1739-1749.

22. Matsumoto T, Rauskolb S, Polack M, et al. Biosynthesis and processing of endogenous BDNF: CNS neurons store and secrete BDNF, not pro-BDNF. Nat Neurosci. 2008;11(2):131-133.

23. Yan Q, Radeke MJ, Matheson CR, Talvenheimo J, Welcher AA, Feinstein SC. Immunocytochemical localization of TrkB in the central nervous system of the adult rat J Comp Neurol. 1997;378(1):135-157.

24. Lu B, Buck CR, Dreyfus CF, Black IB. Expression of NGF and NGF receptor mRNAs in the developing brain: evidence for local delivery and action of NGF. Exp Neurol. 1989;104(3):191-199.

25. Kaplan DR, Miller FD. Neurotrophin signal transduction in the nervous system. Curr Opin Neurobiol. 2000;10(3):381-391.

26. Koshimizu H, Hazama S, Hara T, Ogura A, et al. Distinct signaling pathways of precursor BDNF and mature BDNF in cultured cerebellar granule neurons. Neurosci Lett. 2010;473(3):229-232.

27. Martinowich K, Manji H, Lu B. New insights into BDNF function in depression and anxiety. Nat Neurosci. 2007;10(9):1089-1093.

28. Nagahara AH, Tuszynski MH. Potential therapeutic uses of BDNF in neurological and psychiatric disorders. Nat Rev Drug Discov. 2011;10(3):209-219.

29. Castrén E, Kojima M. Brain-derived neurotrophic factor in mood disorders and antidepressant treatments. Neurobiol Dis. 2017;97(Pt B):119-126.

30. Tripp A, Oh H, Guilloux JP, Martinowich K, et al. Brain-derived neurotrophic factor signaling and subgenual anterior cingulate cortex dysfunction in major depressive disorder. Am J Psychiatry. 2012;169(11):1194-1202.

31. Dwivedi Y, Rizavi HS, Conley RR, Roberts RC, Tamminga CA, Pandey GN. Altered gene expression of brainderived neurotrophic factor and receptor tyrosine kinase $\mathrm{B}$ in postmortem brain of suicide subjects. Arch Gen Psychiatry. 2003;60(8):804-815.

32. Dwivedi Y, Rizavi HS, Zhang H, et al. Neurotrophin receptor activation and expression in human postmortem brain: effect of suicide.Biol Psychiatry. 2009;65(4):319-328. 
(2) 33. Trivedi MH, Rush AJ, Wisniewski SR, et al. Evaluation of outcomes with citalopram for depression using measurement-based care in STAR*D: implications for clinical practice. Am J Psychiatry. 2006;163(1):28-40.

34. Stahl SM. Stahl's essential psychopharmacology: Prescriber's Guide, Sixth Edition. Cambridge: Cambridge University Press.

35. Siuciak JA, Lewis DR, Wiegand SJ, Lindsay RM. Antidepressant-like effect of brain-derived neurotrophic factor (BDNF). Pharmacol Biochem Behav. 1997;56(1):131-137.

36. Schmidt HD, Duman RS. Peripheral BDNF produces antidepressant-like effects in cellular and behavioral models. Neuropsychopharmacology. 2010;35(12):2378-2391.

37. Rantamäki T, Hendolin P, Kankaanpää A, et al. Pharmacologically diverse antidepressants rapidly activate brainderived neurotrophic factor receptor TrkB and induce phospholipase-Cgamma signaling pathways in mouse brain. Neuropsychopharmacology. 2007;32(10):2152-2162.

38. Saarelainen T, Hendolin P, Lucas G, et al. Activation of the TrkB neurotrophin receptor is induced by antidepressant drugs and is required for antidepressant-induced behavioral effects. J Neurosci. 2003;23(1):349-357.

39. Nestler EJ, Carlezon WA Jr. The mesolimbic dopamine reward circuit in depression. Biol Psychiatry. 2006;59(12):1151-1159.

40. Eisch AJ, Bolaños CA, de Wit J, et al. Brain-derived neurotrophic factor in the ventral midbrain-nucleus accumbens pathway: a role in depression. Biol Psychiatry. 2003;54(10):994-1005.

41. Berton O, McClung CA, Dileone RJ, et al. Essential role of BDNF in the mesolimbic dopamine pathway in social defeat stress. Science. 2006;311(5762):864-868.

42. Lippmann M, Bress A, Nemeroff CB, Plotsky PM, Monteggia LM. Long-term behavioural and molecular alterations associated with maternal separation in rats. Eur J Neurosci. 2007;25(10):3091-3098.

43. Ankeny DP, McTigue DM, Guan Z, et al. Pegylated brain-derived neurotrophic factor shows improved distribution into the spinal cord and stimulates locomotor activity and morphological changes after injury. Exp Neurol. 2001;170(1):85-100.

44. Winkler J, Ramirez GA, Kuhn HG, et al. Reversible Schwann cell hyperplasia and sprouting of sensory and sympathetic neurites after intraventricular administration of nerve growth factor. Ann Neurol. 1997;41(1):82-93.

45. Williams LR. Hypophagia is induced by intracerebroventricular administration of nerve growth factor. Exp Neurol. 1991;113(1):31-37.

46. Eriksdotter Jönhagen M, Nordberg A, Amberla K, et al. Intracerebroventricular infusion of nerve growth factor in three patients with Alzheimer's disease. Dement Geriatr Cogn Disord. 1998;9(5):246-257.

47. Isaacson LG, Saffran BN, Crutcher KA. Intracerebral NGF infusion induces hyperinnervation of cerebral blood vessels. Neurobiol Aging. 1990;11(1):51-55.

48. Kordower JH, Palfi S, Chen EY, et al. Clinicopathological findings following intraventricular glial-derived neurotrophic factor treatment in a patient with Parkinson's disease. Ann Neurol. 1999;46(3):419-424.

49. Nutt JG, Burchiel KJ, Comella CL, et al. Randomized, double-blind trial of glial cell line-derived neurotrophic factor (GDNF) in PD. Neurology. 2003;60(1):69-73.

50. Lang AE, Gill S, Patel NK, et al. Randomized controlled trial of intraputamenal glial cell line-derived neurotrophic factor infusion in Parkinson disease. Ann Neurol. 2006;59(3):459-466.

51. Gasmi M, Herzog CD, Brandon EP, et al. Striatal delivery of neurturin by CERE-120, an AAV2 vector for the treatment of dopaminergic neuron degeneration in Parkinson's disease. Mol Ther. 2007;15(1):62-68.

52. Rieder R, Wisniewski PJ, Alderman BL, Campbell SC. Microbes and mental health: A review.Brain Behav Immun. 2017;66:9-17.

53. Cirulli F, Berry A, Chiarotti F, Alleva E. Intrahippocampal administration of BDNF in adult rats affects short-term behavioral plasticity in the Morris water maze and performance in the elevated plus-maze. Hippocampus. 2004;14(7):802-807.

54. Rios M, Fan G, Fekete C, et al. Conditional deletion of brain-derived neurotrophic factor in the postnatal brain leads to obesity and hyperactivity. Mol Endocrinol. 2001;15(10):1748-1757.

55. Koponen E, Võikar V, Riekki R, et al. Transgenic mice overexpressing the full-length neurotrophin receptor trkB exhibit increased activation of the trkB-PLCgamma pathway, reduced anxiety, and facilitated learning. Mol Cell Neurosci. 2004;26(1):166-181.

56. Lang UE, Hellweg R, Kalus P, et al. Association of a functional BDNF polymorphism and anxiety-related personality traits. Psychopharmacology (Berl). 2005;180(1):95-99. 

convection-enhanced delivery of AAV2 vectors [published correction appears in Mol Ther. 2012 Feb;20(2):468. Salegio, Ernesto Aguilar . Mol Ther. 2010;18(8):1490-1495.

58. Goverdhana S, Puntel M, Xiong W, et al. Regulatable gene expression systems for gene therapy applications: progress and future challenges. Mol Ther. 2005;12(2):189-211.

59. Massa SM, Yang T, Xie Y, et al. Small molecule BDNF mimetics activate TrkB signaling and prevent neuronal degeneration in rodents. J Clin Invest. 2010;120(5):1774-1785.

\section{Correspondence:}

Matei Palimariciuc,

M.D., PhD Student, Assistant professor, "Grigore T.Popa” University of Medicine and Pharmacy, Iași; junior psychiatrist, matei.palimariciuc@umfiasi.ro

Submission: 01 aug 2020

Acceptance: 9 sep 2020 